\title{
Humoral Immune Response to COVID-19 Vaccination in Hemodialysis Patients: A Retrospective, Observational Case-Control Pilot Study
}

\author{
Luca Piscitani $^{1} \cdot$ Rita Del Pinto $^{2} \cdot$ Andrea Basili $^{1} \cdot$ Marilena Tunno $^{1} \cdot$ Claudio Ferri $^{2}$
}

Received: 5 December 2021 / Accepted: 22 December 2021 / Published online: 3 January 2022

(C) Italian Society of Hypertension 2021

\begin{abstract}
Introduction Coronavirus 2 disease is associated with increased mortality and morbidity in chronic hemodialysis patients Methods A retrospective, observational case-control pilot study was conducted on consecutive hemodialysis outpatients (cases) and control group of individuals with preserved renal function. Complete SARS-CoV-2 vaccination with BNT162b2 mRNA vaccine, followed by determination of serum antibodies after the second dose, were required from participants in both groups. Previous COVID-19 was an exclusion criterium.

Results 21 hemodialysis patients $(\mathrm{M}: \mathrm{F}=13: 8$, mean age $67.5 \pm 13.4)$ and 16 controls without chronic kidney disease $(\mathrm{M}: \mathrm{F}=4: 12$, mean age $46.8 \pm 12.7)$ were included. Hemodialysis patients had lower mean titers of serum antibodies to the SARS-CoV-2 spike antigen compared with controls (492.39 vs $1901.20 \mathrm{IU} / \mathrm{mL}$, respectively; $\mathrm{p}<0.001$ ), a finding that was confirmed in the age-matched analysis on 18 participants $(580.8$ vs $1836.4 \mathrm{IU} / \mathrm{mL}, \mathrm{p}=0.006)$.

Conclusions This study supports the finding of hyporesponsiveness to mRNA vaccination among hemodialysis patients.
\end{abstract}

Keywords mRNA vaccines $\cdot$ Hemodialysis $\cdot$ COVID-19 $\cdot$ Antibody activity

\section{Introduction}

Hemodialysis patients (HDPs) are at higher risk of developing severe forms of the disease caused by coronavirus-19 $[1,2]$. Lethality in HDPs with coronavirus-19 is estimated to be between 20 and $30 \%$, and there is an increased risk of coronavirus- 2 acute respiratory syndrome (SARS-CoV-2) in these patients [3]. Vaccination plays a role in the current response to the COVID-19 pandemic. Although mRNA vaccines elicit a strong immune response in the general population, immunization rates in immunocompromised patients, including those on hemodialysis, as well as organ transplant recipients, have not been specifically studied in the pivotal mRNA-1273 and BNT16b2 studies [1,2]. Vaccination of hemodialysis patients has been prioritized over the general population because of the high risk of developing serious

Luca Piscitani

lucpis90@ virgilio.it

1 Nephrology and Dialysis Unit, Department of Medicine, S. Salvatore Hospital, Via Vetoio, 67100 L'Aquila, Italy

2 Internal Medicine and Nephrology Unit, Department of Life, Health, and Environmental Sciences, University of L'Aquila, S. Salvatore Hospital, Via Vetoio, 67100 L'Aquila, Italy complications secondary to COVID-19. However, hyporesponsiveness to vaccination has been frequently reported in these patients, such that booster doses are often needed to achieve adequate protection [4]. The uremic syndrome and extracorporeal circulation seem to play a role in disrupting the innate and adaptive immune response through reduced neutrophil and monocyte function, as well as reduced cellmediated and antibody responses.

Thus, our aim was to examine the humoral response to SARS-CoV-2 vaccination in hemodialysis patients compared with a control group with preserved renal function.

\section{Methods}

\subsection{Study Design}

A retrospective, observational case-control pilot study was conducted between May and June 2021 on consecutive hemodialysis outpatients (cases) who were on regular therapeutic follow-up at the Dialysis Unit since at least 1 month and a control group of individuals with preserved renal function (eGFR $>60 \mathrm{ml} / \mathrm{min} / 1.73 \mathrm{~m}^{2}$ ) enrolled among the local health professionals, who had undergone complete 
SARS-CoV-2 vaccination and subsequent monitoring of the relative humoral response as part of standard of care (cases) or local dispositions for healthworkers (controls). To the purpose of monitoring the humoral response to vaccination, venous blood sampling from the upper arm of each participant occurred 30 days after completing the vaccination schedule. Documented previous natural exposure to SARSCoV-2 was an exclusion criterion for participation in the study. Given the exploratory nature of the study, no formal sample size was calculated.

This study was approved by the local IRB (protocol \# 131080/2021).

\subsection{SARS-CoV-2 Vaccination}

For inclusion in the study, complete SARS-CoV-2 vaccination with first and second dose of BNT162b2 mRNA vaccine (Pfizer BioNTech) within the previous month was required from participants in both groups. Pfizer BioNTech vaccine was selected over alternative vaccines based on the Italian normative dispositions on COVID-19 on frail individuals as well as local availability, and the requirement extended to the control group for methodological consistency, All the participants signed an informed consent when receiving the vaccine doses.

\subsection{Humoral Response to SARS-CoV-2 Vaccination}

The humoral response to SARS-CoV-2 vaccination was assessed for all participants in terms of IgG II antibodies against the spike antigen using fluorescence polarization immunoassay (FPIA) $\left(\right.$ Roche $^{\circledR}$ ) at the local Laboratory. The assay tests positive for antibody titers at least equal to $1 \mathrm{IU} /$ $\mathrm{mL}$. The presumed neutralizing antibody titer according to the assay is $80 \mathrm{IU} / \mathrm{mL}$.

\subsection{Statistics}

Demographic and participants' characteristics were evaluated with unpaired $t$ tests for continuous variables (mean \pm standard deviation, $\mathrm{SD})$ and $\chi^{2}$ tests for categorical variables (N, \%). An age-matched analysis was performed to control for age as a potential source of bias. Differences in antibodies titers to SARS-CoV2 between cases and controls were evaluated by Wilcoxon test. A regression analysis was performed to assess the impact of the duration of hemodialysis treatment (months) on antibody titers. Statistical analyses were performed using R (v 4.0.2). Statistical significance was set at $\mathrm{p}<0.05$.

\section{Results}

After applying the prespecified inclusion and exclusion criteria, 21 hemodialysis patients $(61.9 \%$ males $)$ and 16 controls without chronic kidney disease (26.7\% males) were included in the study. Their clinical and demographic characteristics are reported in Table 1. Briefly, hemodialysis patients were older than controls (mean age $67.5 \pm 13.4$ years); $57.1 \%$ had hypertension, $23.8 \%$ had a history of diabetes, $42.9 \%$ had documented peripheral vascular disease and $38.1 \%$ had established cardiovascular disease including previous ischaemic heart disease, stroke, TIA). Among controls, $38.5 \%$ had a history of hypertension; no comorbidities were reported.

Overall, hemodialysis patients had lower mean titers of serum antibodies to the SARS-CoV-2 spike antigen compared with controls (492.39 vs $1901.20 \mathrm{IU} / \mathrm{mL}$, respectively; $\mathrm{p}<0.001$; Fig. 1, panel A). Antibody titers were apparently not affected by the duration of hemodialysis in the examined sample (Fig. 2).

The age-matched analysis on 18 participants, equally distributed between cases and controls (mean age $55.3 \pm 10.5$ years and $55.3 \pm 6.9$ years, respectively; $\mathrm{p}=1.000$; Table 2), confirmed the observation of lower antibody titers among those on hemodialysis (580.8 vs 1836.4 IU/mL, respectively; p < 0.001) (Fig. 1, panel B).
Table 1 Demographic and clinical characteristics of the examined sample

\begin{tabular}{lllr}
\hline & Healthy controls (n. 15) & $\begin{array}{l}\text { Hemodialysis patients } \\
\text { (n. 21) }\end{array}$ & p-value \\
\hline Age, years (mean, SD) & $46.8(12.6)$ & $67.5(13.4)$ & $<0.001$ \\
Men (\%) & $4(26.7)$ & $13(61.9)$ & 0.080 \\
Hypertension (\%) & $5(38.5)$ & $12(57.1)$ & 0.480 \\
Diabetes (\%) & $0(0)$ & $5(23.8)$ & 0.122 \\
PAD (\%) & $0(0)$ & $9(42.9)$ & 0.011 \\
CVD (\%) & $0(0)$ & $8(38.1)$ & 0.021 \\
Ab COV2, IU/mL (mean, SD) & $1901.20(287.33)$ & $492.39(713.09)$ & $<0.001$ \\
\hline
\end{tabular}

$S D$ standard deviation, $P A D$ peripheral artery disease, $C V D$ cardiovascular disease 
A

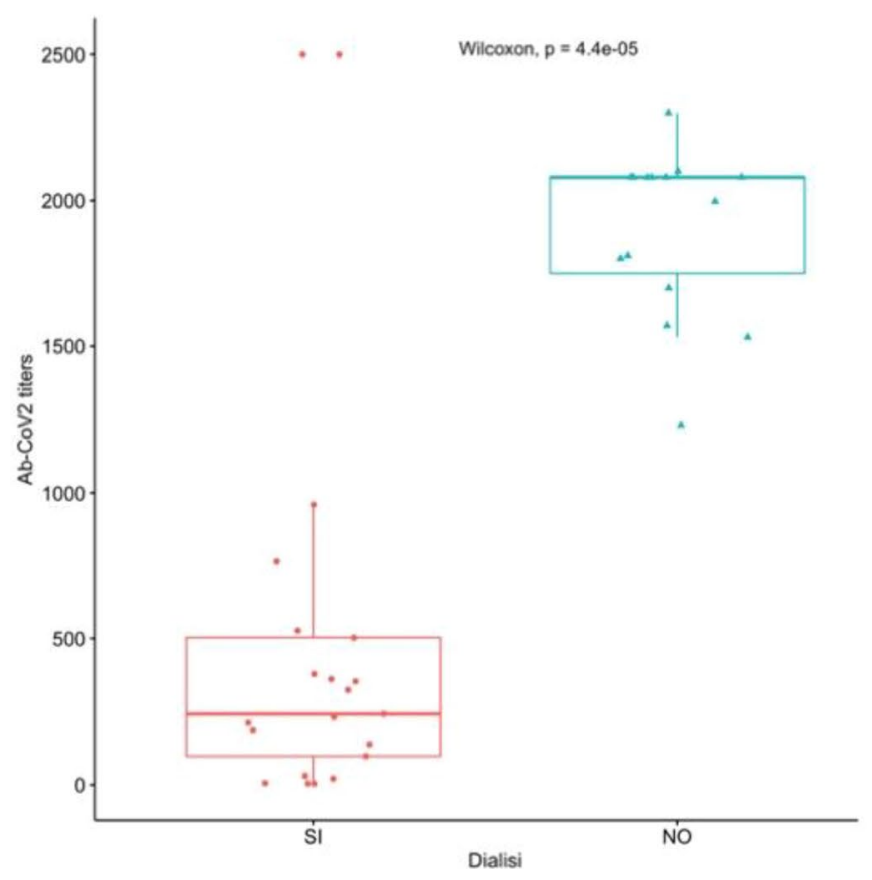

B

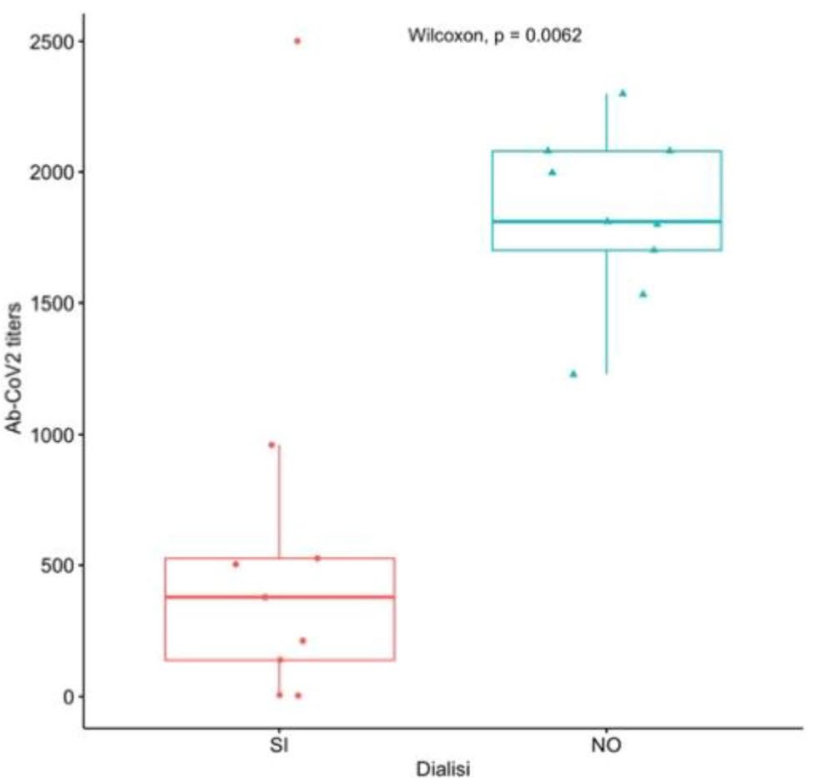

Fig. 1 Boxplots of serum antibodies titers to the SARS-CoV-2 spike antigen in cases and controls. Panel A overall analysis. Panel B agematched analysis

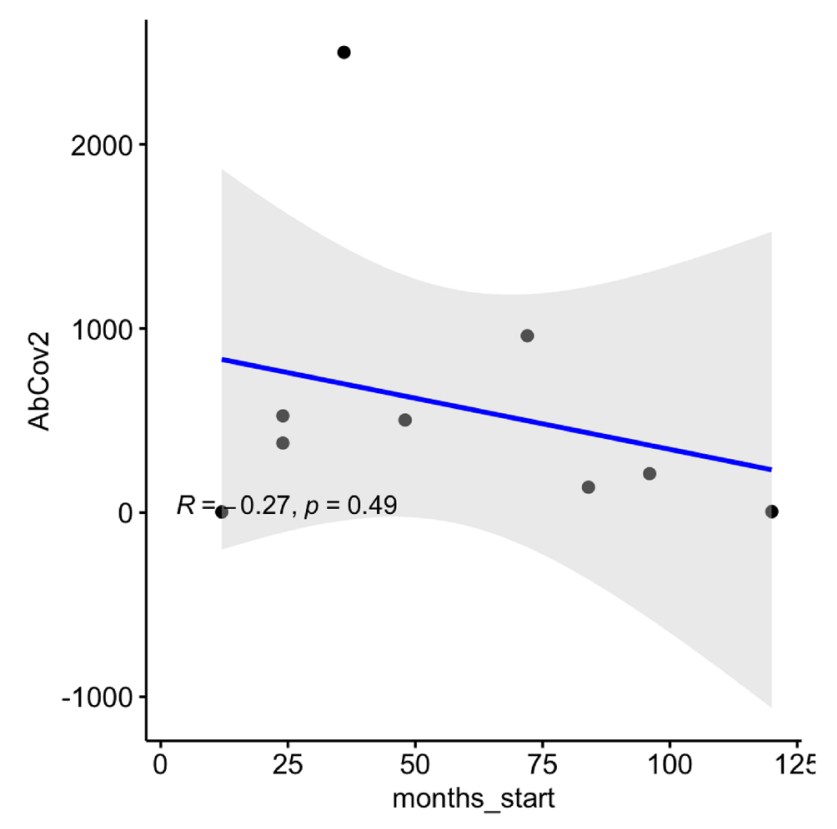

Fig. 2 Regression analysis assessing the impact of hemodialysis duration on antibody titers to the SARS-CoV-2 spike antigen

\section{Discussion}

This retrospective, observational pilot study shows that hemodialysis patients have lower antibody titers against SARS-CoV2 mRNA vaccination compared with healthy controls, possibly reflecting impaired immune competence.

COVID-19 was first described in China in December 2019, and the COVID-19 outbreak was subsequently declared pandemic by the World Health Organization (WHO) (citare). An initial analysis documented that approximately $20 \%$ of HDPs was positive for COVID-19 [5] with frequent clustering of cases among hemodialysis patients HDPs and the medical staff. Conversely, patients performing home dialysis techniques such as peritoneal dialysis and home hemodialysis were relatively protected during this pandemic [6].

UK renal Registry data showed a $20 \%$ mortality rate at 14 days in hemodialysis patients with COVID-19 infection during the first pandemic wave. Such a significantly increased risk was acknowledged in tools such as The Association of Local Authority Medical Advisors (ALAMA) age score and the Q-COVID risk calculator.

Vaccination has, therefore, been declared of primary importance in HDPs. However, the Emergency Use Authorization (EUA) issued by the US Food and Drug Administration (FDA) was based on clinical trials that did not include HDPs or transplant patients. The hyporesponsiveness of these patients 
Table 2 Demographic and clinical characteristics of participants included in the agematched analysis

\begin{tabular}{llll}
\hline & Healthy controls (n. 9) & Hemodialysis patients (n. 9) & p-value \\
\hline Age, years (mean, SD) & $55.3(6.9)$ & $55.3(10.4)$ & 1.000 \\
Men (\%) & $2(22.2)$ & $4(44.4)$ & 0.617 \\
Hypertension (\%) & $5(62.5)$ & $5(55.6)$ & 1.000 \\
Diabetes (\%) & $0(0)$ & $3(33.3)$ & 0.206 \\
PAD (\%) & $0(0)$ & $4(44.4)$ & 0.089 \\
CVD (\%) & $0(0)$ & $2(22.2)$ & 0.453 \\
Ab COV2, IU/mL (mean, SD) & $1836.44(324.23)$ & $580.80(780.49)$ & $<0.001$ \\
Hemodialysis duration, months (men, SD) & - & $3.92(0.36)$ & NA \\
\hline
\end{tabular}

$S D$ standard deviation, $P A D$ peripheral artery disease, $C V D$ cardiovascular disease to vaccination is well known. It has been reported that postinfluenza vaccine seroprotection rates range from 33 to $80 \%$ [7]. The uremic syndrome and extracorporeal circulation seem to play a role in disrupting the innate and adaptive immune response through reduced neutrophil and monocyte function, as well as reduced cell-mediated and antibody responses. Other risk factors that seem to take part in the reduction of defense capabilities are older age, diabetes, time since first dialysis, and malnutrition [8].

Despite the limitation of the small sample size, this study fits together with other works in the analysis of humoral response in hemodialyzed patient undergoing mRNA vaccine. In our study all patients developed a specific humoral response post-vaccination, but the level of antibody production was lower than in control patients without renal disease. Other studies seem to confirm this finding in both HDPs $[9,10]$ and renal transplant patients [11].

\section{Conclusions}

This study demonstrates a hyporesponsiveness of hemodialysis patients to vaccination against COVID-19. This finding in a highly vulnerable population is highly promising. Future studies are required to describe the relationship between antibody titers, functional antibody activity, cell-mediated response, whether antibody titer is predictive of immune protection, and whether there is a need for a booster dose of mRNA vaccines in hemodialysis patients.

\section{Declarations}

Conflict of interest All the authors declare no conflict of interest

Ethical approval The study was approved by the local IRB with protocol n. 131080/2021.

\section{References}

1. Razzaghi H, Wang Y, Lu H, Marshall KE, Dowling NF, PazBailey G, Twentyman ER, Peacock G, Greenlund KJ. Estimated county-level prevalence of selected underlying medical conditions associated with increased risk for severe COVID-19 illness-United States, 2018. MMWR Morb Mortal Wkly Rep. 2020;69(29):945-50. https://doi.org/10.15585/mmwr.mm692 $9 \mathrm{a} 1$.

2. Kliger AS, Silberzweig J. COVID-19 and dialysis patients: unsolved problems in early 2021. J Am Soc Nephrol. 2021;32(5):1018-20. https://doi.org/10.1681/ASN.2020121766.

3. Jager KJ, Kramer A, Chesnaye NC, Couchoud C, Sánchez-Álvarez JE, Garneata L, Collart F, Hemmelder MH, Ambühl P, Kerschbaum J, Legeai C, Del Pino Y, Pino MD, Mircescu G, Mazzoleni L, Hoekstra T, Winzeler R, Mayer G, Stel VS, Wanner C, Zoccali C, Massy ZA. Results from the ERA-EDTA Registry indicate a high mortality due to COVID-19 in dialysis patients and kidney transplant recipients across Europe. Kidney Int. 2020;98(6):15408. https://doi.org/10.1016/j.kint.2020.09.006.

4. Patel N, Assimon MM, Bruni E, McNutt LA, Mason DL. Incidence and clinical predictors of nonresponse to hepatitis B vaccination among patients receiving hemodialysis: importance of obesity. South Med J. 2015;108(9):567-73. https://doi.org/10. 14423/SMJ.0000000000000343.

5. Corbett RW, Blakey S, Nitsch D, Loucaidou M, McLean A, Duncan N, Ashby DR, West London Renal and Transplant Centre. Epidemiology of COVID-19 in an urban dialysis center. J Am Soc Nephrol. 2020;31(8):1815-23. https://doi.org/10.1681/ASN. 2020040534.

6. Truong T, Dittmar M, Ghaffari A, Lin E. Policy and pandemic: the changing practice of nephrology during the coronavirus disease-2019 outbreak. Adv Chronic Kidney Dis. 2020;27(5):390-6. https://doi.org/10.1053/j.ackd.2020.06.003.

7. Krueger KM, Ison MG, Ghossein C. Practical guide to vaccination in all stages of CKD, including patients treated by dialysis or kidney transplant. Am J Kidney Dis. 2020;75:417-25.

8. Kato S, Chmielewski M, Honda H, Pecoits-Filho R, Matsuo S, Yuzawa Y, Tranaeus A, Stenvinkel P, Lindholm B. Aspects of immune dysfunction in end-stage renal disease. Clin J Am Soc Nephrol. 2008;3(5):1526-33. https://doi.org/10.2215/CJN.00950 208. 
9. Jahn M, Korth J, Dorsch O, Anastasiou OE, Sorge-Hädicke B, Tyczynski B, Gäckler A, Witzke O, Dittmer U, Dolff S, Wilde B, Kribben A. Humoral response to SARS-CoV-2-vaccination with BNT162b2 (Pfizer-BioNTech) in patients on hemodialysis. Vaccines (Basel). 2021;9(4):360. https://doi.org/10.3390/vaccines90 40360.

10. Janay NB, Freiman S, Shapira M. Experience with SARS-CoV-2 BNT162b2 mRNA vaccine in dialysis patients. Kidney Int. 2021;99:1487-501.
11. Marion O, Del Bello A, Abravanel F, Couat C, Faguer S, Esposito L, Hebral AL, Izopet J, Kamar N. Safety and immunogenicity of anti-SARS-CoV-2 messenger RNA vaccines in recipients of solid organ transplants. Ann Intern Med. 2021;174(9):1336-8. https:// doi.org/10.7326/M21-1341. 